\title{
Brain Tumour Detection using PSO and Region Growing Algorithm
}

\author{
Rupinder Kaur ${ }^{1}$, Gurjit Singh ${ }^{2}$ \\ M.Tech Scholar, ECE, ACET, Amritsar, India ${ }^{1}$ \\ Assistant Professor, ECE, ACET, Amritsar, India ${ }^{2}$
}

\begin{abstract}
Brain tumor, which is one of the most common brain diseases, has affected and devastated many lives. The statistical reports shows low survival rate of brain tumor patients although brain tumor diseases has been the centre of attention of thousands of researchers over several decades, around the world. In the recent years, researchers from different disciplines ranging from medical to mathematical and computer sciences have combined their knowledge and efforts for better understanding of the disease and to find more effective treatments The proposed algorithm of particle swarm optimization is an evolutionary computational model and is Particle Swarm Optimization belongs to the class of swarm intelligent techniques that are used to solve the Optimization problem in real life. Fragmentation, detection and extraction of the infected tumor region of MRI are the main concern, but arduous task and time taken by radiologist, depends on their experience only
\end{abstract}

Keywords: MRI, X-rays, PSO, Segmentation

\section{INTRODUCTION}

Brain tumor, which is one of the most common brain diseases, has affected and devastated many lives. According to study of International Agency for Research on Cancer (IARC), it is estimated that more than 126,000 people are diagnosed for brain tumor per year around the world. The mortality rate is around 97,000 per year [5]. The statistical reports shows low survival rate of brain tumor patients although brain tumor diseases has been the centre of attention of thousands of researchers over several decades, around the world.[6]In the presence of several medical imaging techniques, Magnetic Resonance Imaging (MRI) technique occupies a predominant position in the area of diagnosing the organs of human body. The functionality of an MRI scanner entirely relies upon the $80 \%$ of hydrogen atom present in our body. The hydrogen atom of our human body is stimulated using resonant radio frequency signals. The excited hydrogen atom is capable of emitting the absorbed radio frequency signals, upon which several signal processing operations are done to obtain a clear anatomy of human organs and tissues.[7]

\section{Nervous system}

The nervous system is divided into central and peripheral systems. The central nervous system (CNS) is composed of the brain and spinal cord. The peripheral nervous system (PNS) is composed of spinal nerves that branch from the spinal cord and cranial nerves that branch from the brain. The PNS includes the autonomic nervous system, which controls vital functions such as breathing, digestion, heart rate, and secretion of hormones.

Skull The purpose of the bony skull is to protect the brain from injury. The skull is formed from 8 bones that fuse together along suture lines. These bones include the frontal, parietal (2), temporal (2), sphenoid, occipital and ethmoid (Fig. 1). The face is formed from 14 paired bones including the maxilla ,zygoma, nasal, palatine, lacrimal, inferior nasal conches, mandible, and vomer.[8]

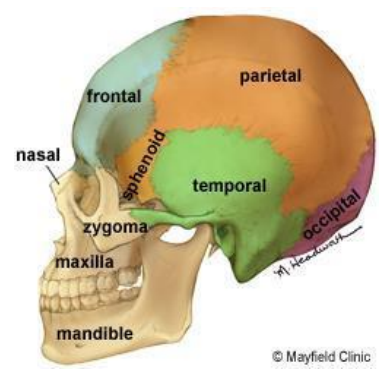

Figure1 Eight bones form the skull and fourteen bones form the face [12] 
Vol. 8, Issue 2, February 2019

\section{IMAGE PROCESSING}

Image processing is a methodology which is capable of converting an image into digital form and it performs certain operations on image, so as to achieve an enhanced image or to extract some vital information from it. It is similar to digital signal processing. In image processing, input is an image (may be a video frame or a photograph in any format) and the output may be an image or the characteristics of the input image. Image processing system usually considers an image as a two dimensional signal, while processing. It is one among the emerging technologies, with its branches of application widespread into several domains of business. Image processing is a core research area in engineering and it also acts as a thrust area in other disciplines of computer science. Researchers are in need of image processing; as it offers real time applications and the results derived from image processing techniques are also made available to the hands of its user.[10]

There are two main methods in image processing and they are:

1. Analog Image Processing

2. Digital Image Processing

\section{LITERATURE REVIEW}

Sunitha Set al. [2014] [1] - Manually detecting and segmenting brain tumors from brain MRI, in cases where a large number of MRI scans are taken for each patient, is tedious and subjected to inter and intra observer detection and segmentation variability. Therefore, there is a need for computer aided brain tumor detection and segmentation from brain MR images to overcome the tedium and observer variability involved in the manual segmentation. A number of methods have been proposed in recent years to fill this gap, but still there is no commonly accepted automated technique for use in clinical floor by clinicians due to accuracy and robustness issues. This paper, presents a review of the methods used for MRI brain tumor segmentation. The review covers imaging modalities, magnetic resonance imaging and methods for noise reduction and segmentation approaches.

RituRana et al. [2015] [2] - A brain tumor is an abnormal growth of tissue in the brain or central spine that can disrupt proper brain function and creates an increasing pressure in the brain. This paper is intended to present a comprehensive review of the methods of brain tumor detection through Magnetic Resonance Imaging (MRI) technique used in different stages of Computer Aided Detection System (CAD). It also provides a brief background on brain tumor in general and non-invasive imaging of brain tumor in order to give a comprehensive insight into the field. Lastly, the paper concludes with a concise discussion and provides a direction toward the upcoming trend of more advanced research studies on brain image segmentation and tumor detection.

NehaRaniet al. [2016] [3] - Brain is an organ that controls activities of all the parts of the body. Recognition of automated brain tumor in Magnetic resonance imaging (MRI) is a difficult task due to complexity of size and location variability. This automatic method detects all the type of cancer present in the body. Previous methods for tumor are time consuming and less accurate. In the present work, statistical analysis morphological and threes holding techniques are used to process the images obtained by MRI. Feed-forward back-prop neural network is used to classify the performance of tumors part of the image. This method results high accuracy and less iterations detection which further reduces the consumption time.

Riddhi.S.Kapse et al. [2015] [4] - : Today's recent medical imaging research faces the challenge of detecting brain tumor through Magnetic Resonance Images (MRI). Broadly, to produce images of soft tissue of human body, MRI images are used by experts. For brain tumor detection, image segmentation is required. Mechanizing this process is a tricky task because of the high diversity in the appearance of tumor tissues among different patients and in many cases similarity with the usual tissues

\section{III.PROPOSED METHODOLOGY}

\section{PROPOSED METHODOLOGY}

Image segmentation procedure adopted to analyze medical images is quite a challenging task. It refers to the process of partitioning a digital image into multiple regions. The goal of segmentation is to change the representation of an image into something that is more meaningful and easier to analyze. Image segmentation is used to locate objects and boundaries in images. The result of image segmentation occurs as a set of regions that collectively covers the entire image. The algorithm of particle swarm optimization is an evolutionary computational model and is Particle Swarm Optimization belongs to the class of swarm intelligent techniques that are used to solve the Optimization problem in real life itself .PSO simulates the behavior of bird flocking. Means, a group of bird finding the food randomly in an area. There is only one piece of food in the area being searched by all the birds. They do not know where food is is, but they know how far the food is in each iteration. So the best way to find the food is to follow the bird which is nearest to the food. Flocking behavior is exhibited when group of birds are foraging 
Vol. 8, Issue 2, February 2019

Each particle in PSO is updated by two best values

pbest- Each particle keeps track of its coordinates in the solution space which are associated with the bestsolution (fitness) that has achieved so far by that particle. This value is called personal best, pbest.

gbest - It is tracked by the PSO is the best value obtained so far by any particle in the neighbourhood of thatparticle. This value is called Global Best, gbest.

\section{REGION GROWING ALGORITHM:}

The first region growing method was the seeded region growing method. This method task was to take a set of seeds as input along with the image. The seed mark each of the object to be segmented the region are grown by comparing all unallocated neighborhood pixels to the regions .the difference between a pixel density value and the region mean ,is used as a measure of similarity .the pixel with the smallest difference measured this way is allocated to the respective regions. the two main approaches are "Bottom-up" region growing and secondly "top bottom " region splitting and merging approach but we will go for first approach.

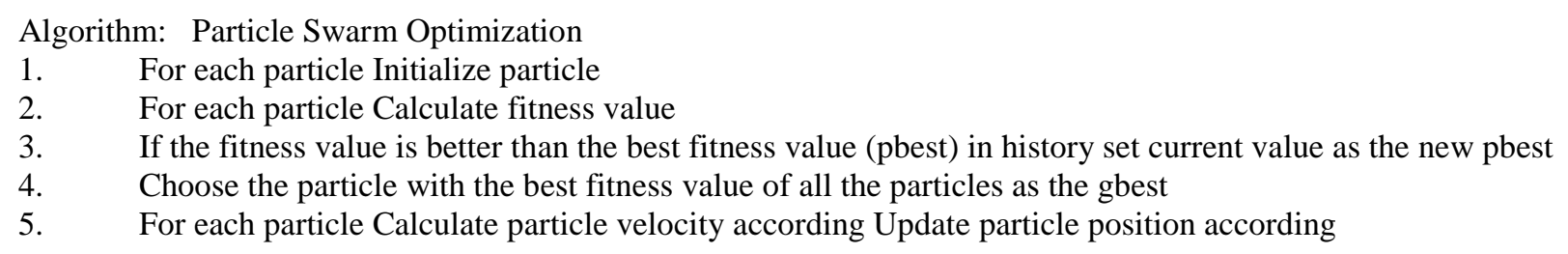

$$
v_{i d}^{\text {best }}=w * v_{i d}^{\text {pre }}+c_{1} * \operatorname{rand} *\left(p_{i d}^{\text {best }}-x_{i d}\right)+c_{2} * \operatorname{rand}() *\left(p_{\text {aid }}^{\text {best }}-x_{i d .}\right)
$$$$
p_{i d}^{\text {new }}=p_{i d}^{\text {pre }}+v_{i d}^{\text {new }}
$$

Where, vid is the velocity of particle in the $d$-dimension search space and is limited into [Vmin, Vmax]. Vmin and Vmaxare respectively, the minimum and maximum the moving distance in one-step, pidis the position ofithparticle $i$, pidbest is the individual best position of particle $I$, pgidbest is the gidth global best position in all particles, xid is the current position of particle $i$, rand () is a random number function and its value are between 0 and 1 , w is the inertia weight, $\mathrm{c} 1$ and $\mathrm{c} 2$ are constants

REGION GROWING ALGORITHM: The first region growing method was the seeded region growing method. This method task was to take a set of seeds as input along with the image. The seed mark each of the object to be segmented .the region are grown by comparing all unallocated neighborhood pixels to the regions .the difference between a pixel density value and the region mean, is used as a measure of similarity .the pixel with the smallest difference measured this way is allocated to the respective regions. the two main approaches are "Bottom-up" region growing and secondly "top bottom " region splitting and merging approach but we will go for first approach.

\section{ALGORITHM}

1. Start from seed point.

2. look at its neighborhood.

3. for each point $\mathrm{p}$ in nhood.

4. $\quad$ if $P(p)=$ true then include pin region $L$.

$5 . \quad$ else $\mathrm{p}$ is the border.

6. in each iteration, look at the neighbor of each point in L,to save computation ,"order" the point in the L and don't check the points that have high score (e.g. the oncs that are in the middle of the region).

\section{ALGORITHM FOR COMBINED PSO AND REGION GROWING ALGORITHM}

Step 1:- MR brain images of different pixel size are fed as input to the proposed methodology.

Step 2:- MR brain images with different dimensions and sizes are used as input, say $256 \times 256,480 \times 375,1105 \times 650$ and $763 \times 664$. To standardize the segmentation procedure, pre - processing steps involving image resizing (Conversion to $512 \times 512$ pixel size), RGB (Red Green Blue) to grayscale conversion, skull stripping and patient detail removal are done. Adjustment of image intensity or color map values is performed to handle medical information losses aroused due to image resizing. 
Vol. 8, Issue 2, February 2019

Step 3:-To identify the best or the optimum cluster value using PSOalgorithm.

Step 4:-The best known position of the pixel along with the clusters found using PSO, acts as a leader or the centroid value for Region growing algorithm.

Step 5:-Region growing algorithm is associated with grouping of pixels in a cluster with the centroid value. The clusters are grouped around the centroid value found using PSO algorithm.

Step 6:-The process of grouping the pixels of each cluster with the centroid value takes place for nearly $\mathrm{K}$ number of iterations,

Step 7:-Output image with the tissues of brain segmented and the tumor region identified is obtained from the proposed algorithm.

\section{Experimental Results}

1. Accuracy

58 yr Male Accuracy Comparison

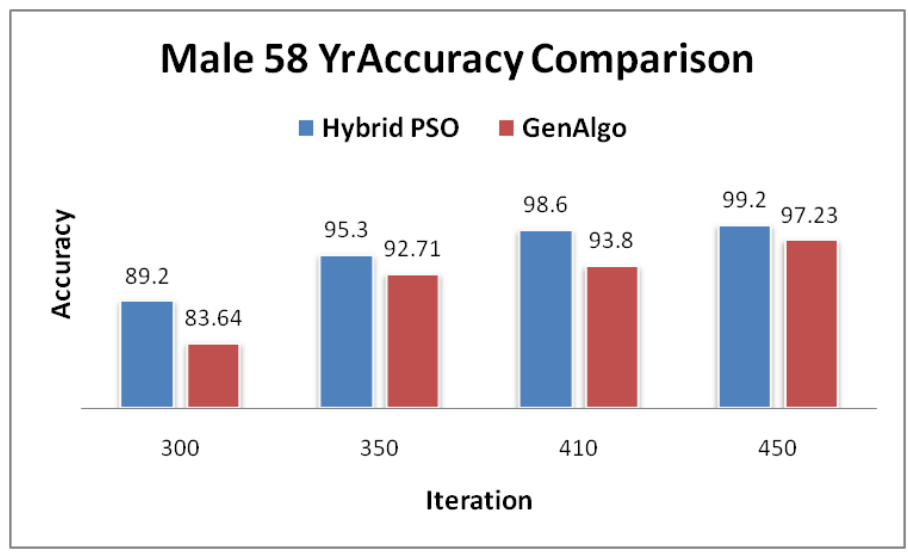

\begin{tabular}{|c|c|c|}
\hline \multicolumn{3}{|c|}{ Male 58 yr Accuracy Comparison } \\
\hline Iteration & Hybrid PSO & GenAlgo \\
\hline 300 & 89.2 & 83.64 \\
\hline 350 & 95.3 & 92.71 \\
\hline 410 & 98.6 & 93.8 \\
\hline 450 & 99.2 & 97.23 \\
\hline
\end{tabular}

Table Male 58 yr Accuracy Comparison

Fig Male 58 yr Accuracy Comparison

The above Fig and table Shows Proposed system shows comparison results of Accuracy on 4 yr Male patient data the System uses Hybrid PSO Algorithm for Brain Tumour Detection the various iteration shows in comparison of genetic result the proposed system shows better results as compared to previous result which shows proposed technique has better result as compared to proposed technique

4yr Male Accuracy Comparison

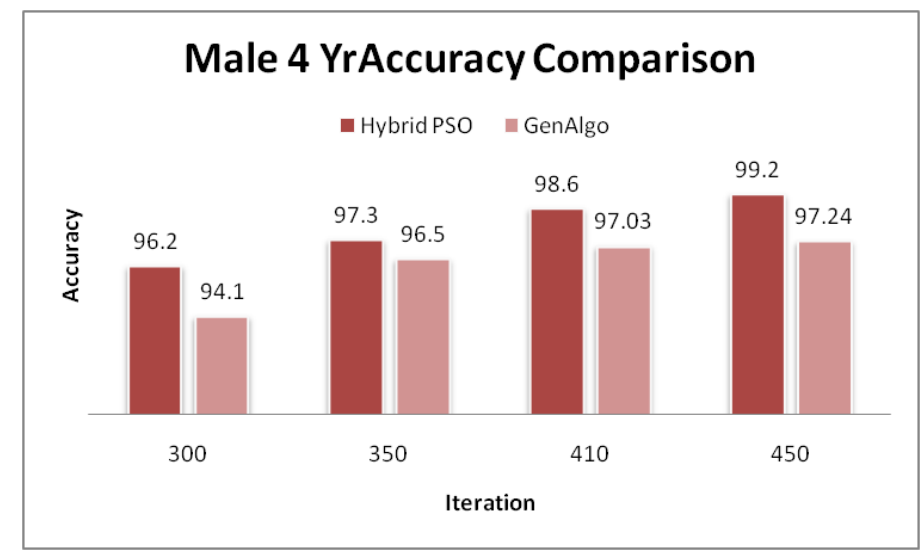

\begin{tabular}{|c|c|c|}
\hline \multicolumn{3}{|c|}{ Male 4 yr Accuracy Comparison } \\
\hline Iteration & Hybrid PSO & GenAlgo \\
\hline 300 & 96.2 & 94.1 \\
\hline 350 & 97.3 & 96.5 \\
\hline 410 & 98.6 & 97.03 \\
\hline 450 & 99.2 & 97.24 \\
\hline
\end{tabular}

Table Male 4yr Accuracy Comparison

Fig Male 4yr Accuracy Comparison

The above Fig and table Shows Proposed system shows comparison results of Accuracy on58yr Male patient data the System uses Hybrid PSO Algorithm for Brain Tumour Detection the various iteration shows in comparison of genetic result the proposed system shows better results as compared to previous result which shows proposed technique has better result as compared to proposed technique 


\section{Execution Time:}

1. 4yr Male Execution Time Comparison

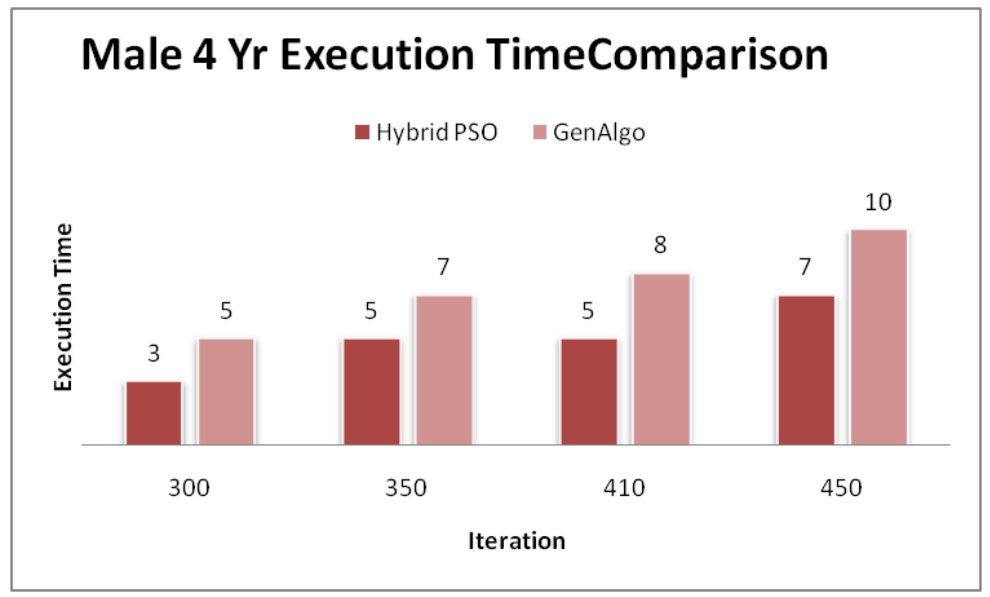

Fig 4 Male Execution Time Comparison

\begin{tabular}{|l|l|l|}
\hline \multicolumn{2}{|l|}{4 Yr Male Execution time Comparison } \\
\hline Iteration & Hybrid PSO & GenAlgo \\
\hline 300 & 3 & 5 \\
\hline 350 & 5 & 7 \\
\hline 410 & 5 & 8 \\
\hline 450 & 7 & 10 \\
\hline
\end{tabular}

Table 4yr Male Execution Time

Comparison

The above Fig and table Shows Proposed system shows comparison results of Execution Time on 4 yr Male patient data the System uses Hybrid PSO Algorithm for Brain Tumor Detection the various iteration shows in comparison of genetic result the proposed system shows better results as compared to previous result which shows proposed technique has better result as compared to proposed technique

\subsection{8yr Male Execution Time Comparison}

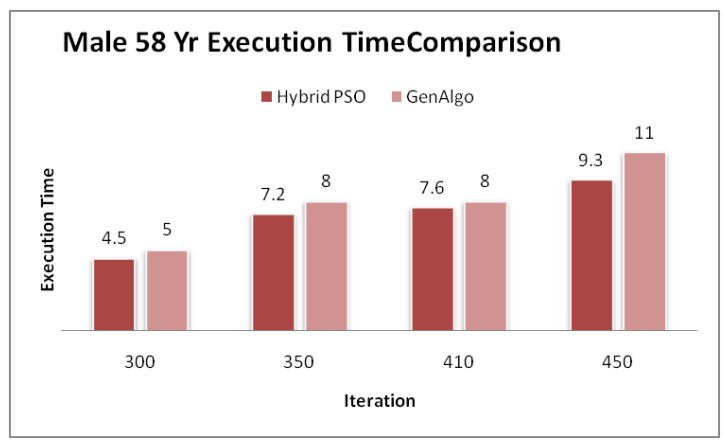

Fig 58 Male Execution Time Comparison

\begin{tabular}{|c|c|c|}
\hline \multicolumn{3}{|c|}{$\begin{array}{l}\text { 58Yr Male Execution time } \\
\text { Comparison }\end{array}$} \\
\hline Iteration & Hybrid PSO & GenAlgo \\
\hline 300 & 4.5 & 5 \\
\hline 350 & 7.2 & 8 \\
\hline 410 & 7.6 & 8 \\
\hline 450 & 9.3 & 11 \\
\hline
\end{tabular}

Table 58yr Male Execution Time

Comparison

The above Fig and table Shows Proposed system shows comparison results of Execution Time on 58 yr Male patient data the System uses Hybrid PSO Algorithm for Brain Tumor Detection the various iteration shows in comparison of genetic result the proposed system shows better results as compared to previous result which shows proposed technique has better result as compared to proposed technique

\section{Stability:}

\section{4 yr Stability Comparison}


Vol. 8, Issue 2, February 2019

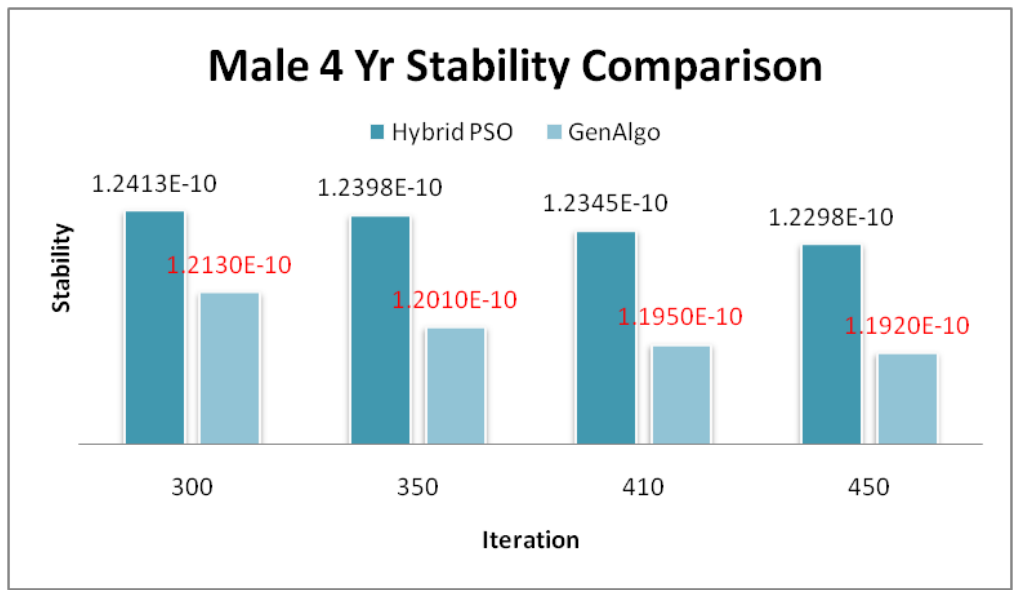

\begin{tabular}{|l|l|l|}
\hline \multicolumn{3}{|c|}{ 4yr Male Stability Comparison } \\
\hline Iteration & $\begin{array}{l}\text { Hybrid } \\
\text { PSO }\end{array}$ & GenAlgo \\
\hline 300 & $1.2413 \mathrm{E}-10$ & $1.2130 \mathrm{E}-10$ \\
\hline 350 & $1.2398 \mathrm{E}-10$ & $1.2010 \mathrm{E}-10$ \\
\hline 410 & $1.2345 \mathrm{E}-10$ & $1.1950 \mathrm{E}-10$ \\
\hline 450 & $1.2298 \mathrm{E}-10$ & $1.1920 \mathrm{E}-10$ \\
\hline \multicolumn{3}{|c|}{ Table 4yr Stability Comparison }
\end{tabular}

Fig 4yr Stability Comparison

The above Fig and table Shows Proposed system shows comparison results of Stability on 4 yr Male patient data the System uses Hybrid PSO Algorithm for Brain Tumor Detection the various iteration shows in comparison of genetic result the proposed system shows better results as compared to previous result which shows proposed technique has better result as compared to proposed technique

1. 58 yr Stability Comparison

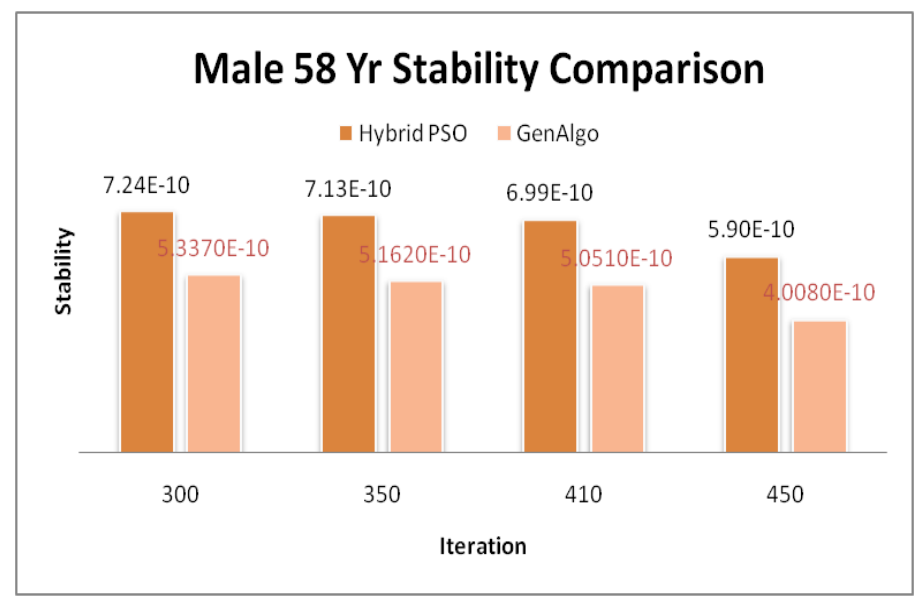

\begin{tabular}{|c|l|l|}
\hline \multicolumn{3}{|c|}{ 58yr Male Stability Comparison } \\
\hline Iteration & $\begin{array}{l}\text { Hybrid } \\
\text { PSO }\end{array}$ & GenAlgo \\
\hline 300 & $7.24 \mathrm{E}-10$ & $5.3370 \mathrm{E}-10$ \\
\hline 350 & $7.13 \mathrm{E}-10$ & $5.1620 \mathrm{E}-10$ \\
\hline 410 & $6.99 \mathrm{E}-10$ & $5.0510 \mathrm{E}-10$ \\
\hline 450 & $5.90 \mathrm{E}-10$ & $4.0080 \mathrm{E}-10$ \\
\hline
\end{tabular}

Table 58yr Stability Comparison

Fig 58yr Stability Comparison

The above Fig and table Shows Proposed system shows comparison results of Stability on 58 yr Male patient data the System uses Hybrid PSO Algorithm for Brain Tumor Detection the various iteration shows in comparison of genetic result the proposed system shows better results as compared to previous result which shows proposed technique has better result as compared to proposed technique

\section{IV.CONCLUSION}

PSO with region growing algorithm is proposed to classify magnetic resonance images. It is suggested that the use of the growing region algorithm classifies magnetic resonance images. The improved outcome is obtained from proposed algorithm; we must carefully choose the different values of the standards of peace monitoring. The recognition of the tumor is evaluated by performance measures, namely the results of this method demonstrate that the advanced results with a growing approach in the region have better accuracy in the classification. 


\section{REFERENCES}

[1] Sunitha S and SanthoshiSukumaran, "A Review on Brain Tumor Segmentation”, International Journal of Engineering Research \& Technology (IJERT), Vol. 3, ISSN: 2278-0181, ,pp.332-335, April - 2014

[2] RituRana and Parvinder Singh, "Brain Tumor Detection through MR Images: A Review of Literature", IOSR Journal of Computer Engineering (IOSR-JCE), Vol. 17, ISSN: 2278-8727, pp.7-18, 2015.

[3] Neha Rani and ShardaVashisth, "Brain Tumor Detection and Classification with Feed Forward Back-Prop Neural Network", International Journal of Computer, Volume 12, pp.1-6, July 2016.

[4] Riddhi.S.Kapse, Dr. S.S. Salankar and Madhuri.Babar, "Literature Survey on Detection of Brain Tumor from MRI Images IOSR Journal of Electronics and Communication Engineering (IOSR-JECE), Volume 10, ISSN: 2278-2834, PP 80-86, 2015.

[5] Pragati G. Patil1, Dr. K. J. Karande2, "Brain tumor detection techniques: A Survey", International Research Journal of Engineering and Technology (IRJET), Vol. 3, ISSN: 2395 -0056, pp.396-399, April - 2016.

[6] Nikita Singh1 and Naveen Choudhary2, "A Survey: Brain tumor detection techniques of Computer aided diagnosis through MRI image", International Journal of Engineering Research \& Technology (IJERT), Vol. 6, ISSN: 1694-0814, pp.148-157, April - 2015.

[7] AmitVerma* and GayatriKhanna, "A Survey on Digital Image Processing Techniques for Tumor Detection Indian Journal of Science and Technology, Vol 9, ISSN (Print) : 0974-6846, April 2016

[8] 1Simran Arora*, 2Gurjit Singh, "A Study of Brain Tumor Detection "International Journal of Advanced Research in Computer Science and Software Engineering, Vol. 5, ISSN: 2277 128X, pp.1272-1278, April - 2015.

[9] Rajeev Kumar and Dr. K. James Mathai, "Critical Survey of Different Clustering Algorithm for Effective Tumor Detection", International Journal on Recent and Innovation Trends in Computing and Communication, Vol. 5, ISSN: 2321-8169, pp.896-899, 2017.

[10] A.Sindhu1, S.Meera2, “A Survey on Detecting Brain Tumorinmri Images Using Image Processing Techniques",International Journal of Innovative Research in Computer and Communication Engineering, Vol. 3, ISSN: 2320-9801, pp.123-129, April - 2015.

[11] M .Queen, T.M. BabiMol M.E, “A Survey on Brain Tumor Classification Using Artificial Neural Network”,International Journal of Advanced Research in Computer Engineering \& Technology (IJARCET), Volume 3, pp.3653-3657, November 2014.

[12]S. Cagnoni, A. B. Dobrzeniecki, R. Poli, J. C.Yanch. "Genetic algorithm-based interactive segmentation of 3D medical images", 1999. 